\title{
Warrick's Principle, Implied Linkages and the Effect of Hand/Control Location
}

\author{
Errol R. Hoffmann*
}

\begin{abstract}
Department of Industrial Engineering and Logistics Management, Hong Kong University of Science and Technology, Clearwater Bay, Hong Kong
\end{abstract}

\begin{abstract}
In two-dimensional display/control relationships, Warrick's principle has been found to be an important factor in determining stereotype strengths. This principle has an 'implied linkage' that may bias the responses of persons such as engineers who have knowledge of mechanisms and hence produce stereotypes that are not representative of the general population. It is shown that such 'implied linkages' may also exist for three-dimensional display/control relationships, where the control is not in the same plane as the display. A further factor in such cases may be a 'hand/control location' (HCL) effect that is a physically-based factor related to the location of the control relative to the body, and hence to handedness, hand used and hand posture used in controlling. Several examples show the validity of this concept for both two- and three-dimensional display-control relationships.
\end{abstract}

\section{INTRODUCTION}

An important aspect of designing a device where there is a control that moves an indicator on a display is that the relationship between the control movement and the corresponding movement on the display is 'compatible'. Compatibility is based on the person's expectation about the way in which a device operates. If a large part of the population has the same expectancy about the way in which the control/display relationship works, we say that there is a stereotype for the control/display linkage and the relationship between control and display is compatible.

The purpose of this paper is twofold: firstly to show that there are possibly implied linkages in the case of threedimensional display/control relationships and, secondly, to postulate the existence of a hand/hand-posture effect in determining the stereotypes for three-dimensional displaycontrol relationships.

Warrick's principle [1] has been associated with the mental model of subjects relating control movement to display movement. Warrick's principle can be stated as: 'The indicator is expected to move in the same direction as that part of the control nearest to the display'. This principle, where it is applicable, has been found to be a major contributor to the stereotype found for control movements with two-dimensional display/control relationships. Unfortunately for the determination of population stereotypes, the contribution of the Warrick principle (W) has been found to be dependent on the user population of the device. For example, Hoffmann [2] found that, for engineering students using a two-dimensional control/ display arrangement with both the control and display on the same front face of the device (Fig. 1), the component strength of W was .286 for directional indicators, and .258 for neutral indicators.

*Address correspondence to this author at P.O. Box 89, Halls Gap, Victoria 3381, Australia; E-mail: erroldot@netconnect.com.au

1875-9343/09
The corresponding figures for a group of psychology students were .086 and .060 . Thus for engineering students it contributed an average of $57 \%$ of the total stereotype strength, while for psychology students it contributed just $15 \%$.

It has been suggested that the difference may be due to the knowledge of engineering students about mechanical linkages and hence there was an 'implied linkage' that biased their results to strengthen the Warrick Principle. The group of psychology students had a similar strength of overall stereotype, but this was made up largely of the contribution of the clockwise-to-the-right (CR) principle. The CR principle contributed $39 \%$ to the overall stereotype for engineering students and $83 \%$ for psychology students.

The two-dimensional Warrick 'implied linkage' can be illustrated as a rack and pinion mechanism, with the pinion attached to the control and the rack attached to the indicator, as shown in Fig. (1). Discussion with engineering students has confirmed that they are aware of this linkage when deciding on direction of turn for different control/display relationships.

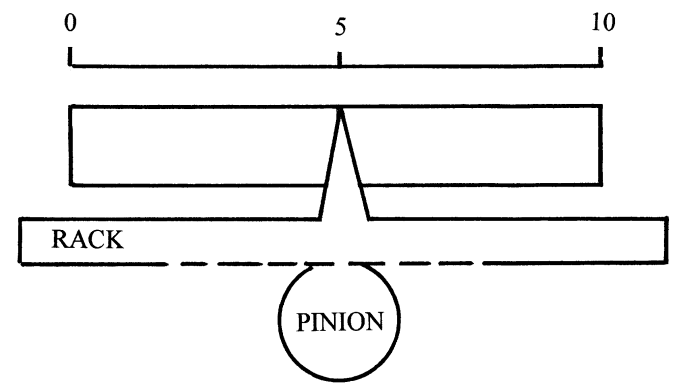

Fig. (1). The 'implied linkage' for two-dimensional display/control relationship where Warrick's principle is operational (linear display with rotary control). 
A consequence of this engineering bias is seen in the cases of a two-dimensional linear display with a control either on the bottom or top of the display (Fig. 2a, b). In case (a), the $\mathrm{W}$ and $\mathrm{CR}$ principles reinforce and hence a strong stereotype is obtained. Engineering students produced a stereotype strength of .95 and Psychology students 1.0 [2]. In case (b) W opposes CR and here Psychology students produced a strong stereotype (.86) due to the weak effect of $\mathrm{W}$ while engineering students produced a weak stereotype (.57) as their strong $\mathrm{W}$ component opposed the CR principle, due to their mental model of an 'implied linkage'.

(a)

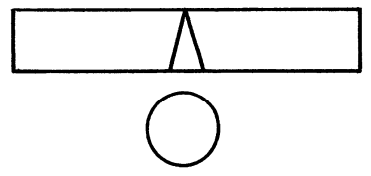

(b)

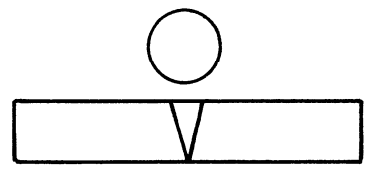

Fig. (2). Cases of two-dimensional display/control relationships where in (a) engineers and psychologists give strong stereotypes and (b) where psychologists have a strong stereotype and engineers a weak stereotype [2].

When there is the possibility of a commonly understood implied linkage among subjects with an engineering background, there may be biases introduced into the data. As much of the recent reported research has been done by academic engineers, and hence engineering students are the most convenient source of subjects, nearly all reported data have come from using these students as subjects. Thus it would appear necessary to be careful in the application of these data and repeat the work using a more general population of subjects. This is of particular importance where a product is to be used by a non-engineering consumer group.

It will be shown in the following that there is a second possibility in three-dimensional display/control relationships that mimics the effect of the Warrick principle in simple cases and which predicts the same component stereotype strengths. It is in more complex cases that this alternative becomes different to the Warrick principle and hence may not have the biasing effect found in simpler cases. This effect is a 'hand/control location' (HCL) term that must be included in the expressions for component strengths. This term is not a stereotype principle in the same way as the Warrick (W), Scale Side (SS, Brebner and Sandow, [3]), Clockwise to Clockwise (CC) and Clockwise to Right (CR) principles [2], which directly relate a control movement to a corresponding display movement. Rather, it is a physical attribute related to the 'natural' rotation of the hand when grasping a control. This 'natural' rotation will be dependent on the hand used (ie a right-hander using the left or right hand), handedness of the operator (as this will determine the 'natural' direction of rotation) and the posture placed on the hand/fingers by the location of the hand relative to the control. The HCL effect is postulated to arise from the biomechanics of the hand/wrist that allows a more comfortable, larger degree of rotation than the opposite direction, when rotating in the direction of movement of the thumb towards the index finger.

Such a physical effect has been studied extensively by Worringham and Berringer [4,5]. In their work the subject was seated in various locations relative to a lever control in order to determine the visual and physical location factors that determined the stereotype of the linear lever movement to the display movement. The hand/control location effect postulated here is different to that studied by Worringham and Berringer in that the display is always in the same location relative to the subject, but the location of the control is changed, requiring the subject to use a different hand or to use the same hand in a different posture.

\section{POSTULATION OF A HAND/CONTROL LOCATION EFFECT}

\section{(a) Two-Dimensional Display-Control Relationships}

For the present case, there is some information available for two-dimensional display/control relationships from studies performed using the preferred and non-preferred hands of right-handed persons and from studies with left and right-handers. This arises from differences between clockwise responses when preferred and non-preferred hands are used (of right handed subjects). Verhaegen et al. [6] studied the stereotypes of 13 groups of mainly African subjects. The data from this paper have been averaged across the 13 groups with the following results:

(i) Rotation of a knob using the right and left hands (no display) in a preferred direction.

When asked to simply rotate a knob using the right or left hands, the proportions of clockwise responses produced were .726 for the right hand and .518 for the left hand. There is thus seen to be a significant effect (Binomial test of proportions, $\mathrm{Z}=6.47, \mathrm{p}<.001$ ). It is suggested that, in the absence of a display, this is evidence of the existence of the HCL effect, showing a distinct preference for a rotation in the direction of pointing of the thumb towards the first finger.

(ii) Use of the left or right hand in responding to case (a) of Fig. (2).

Here the proportion of clockwise responses were .748 and .729 for the right and left hand, respectively. These proportions are not significantly different (Binomial test of proportions, $\mathrm{p}=.085$ ).

(iii) Use of the right and left hands when responding to a vertical display with a side knob location (control and display on the same plane). The proportion of clockwise responses were .746 and .673 for right and left hand use, respectively. These proportions are significantly different $\mathrm{Z}=2.45, \mathrm{p}<.01$ ).

These data show that there is an effect of the hand used, causing, through a difference in the 'natural' direction of rotation of the hand/wrist, a difference in the stereotype. This is the simplest case of an HCL effect. It is shown in a preferred direction of rotation (which is the basic hypothesis of the HCL model) as well as in responses to actual displays. 
In the case where there is a display present, there will be a conflict between the Warrick and HCL components when different hands are used - the components in this case are reinforcing when the right hand is used and in opposition when the left hand is used. However, when there is a display present, the effect of HCL is smaller than without the display present as HCL now competes with the Warrick principle in these two cases.

Data for left and right-handers is fairly limited. Bradley [7] had subjects who were left or right handed respond to a series of instructions related to changing the brightness of a light. In these tests, the direction of functional change was not specified. Of the 30 right-handed subjects, 90\% turned the control knob clockwise. Of 30 left-handed subjects, only $63 \%$ turned the knob clockwise. The difference in responses was significant at $p<.05$. In the general population there is a strong stereotype of clockwise for increasing brightness. In this case the HCL for the right-handers reinforces the clockwise-to-increase component of the stereotype; in the case of left-handers, the components are in opposition and hence the clockwise responses are fewer.

Holding [8] found results similar to the above; the tendency to turn clockwise for an increase was markedly less in left-handed subjects, but where there was a strong established stereotype, this effect was not present [9]. In the seven three-dimensional cases tested [8], where the right hand was used, there was a tendency for clockwise rotation to be used for an increase on the scale reading; in the two cases where the left hand had to be used, this tendency was not present. It should be added that in none of the cases studied was the Warrick principle in its 'unfolded' form applicable. Thus again it seems that there was a hand effect that is compatible with the HCL concept.

Chapanis and Gropper [10] tested a group of 64 subjects who were left or right-handed using their left and right hands. They investigated stereotypes for horizontal and vertical displays where the control was always located below the display (two-dimensional). The authors used two sets of linkages between control and display: one linkage produced an indicator movement to the right or up for a clockwise rotation of the control; the other produced a left or down movement of the indicator for a clockwise control movement. These arrangements were in the direction of, or opposed to, the common stereotype for these control/display relationships. The data for percent of clockwise responses in initial direction of rotation of the control, for the case of the common stereotype, are shown in Fig. (3) for vertical and horizontal displays. Data for the movements in the nonstereotypical direction are not considered.

It is seen that there is a handedness and hand-used effect in these data. The effects may be summarised as follows (quoting from [10]):

$$
\begin{aligned}
& \text { "Consider first the data obtained with the preferred } \\
& \text { hands. When the scale increased in accordance with } \\
& \text { population stereotypes, that is, to the right or upward, } \\
& \text { the right-handed subjects did better than the left- } \\
& \text { handed ones". } \\
& \text { "The data obtained with the non-preferred hands } \\
& \text { show a different pattern. ... the left-handed subjects } \\
& \text { performed better with their non-preferred hands than } \\
& \text { the right-handed subjects did with their non-preferred } \\
& \text { hands on either kind of scale". }
\end{aligned}
$$

The data of (i) above are consistent with the HCL hypothesis. There are possible conflicts with the strongest component stereotype ('clockwise to the right + Warrick' for the horizontal display and 'clockwise for up + scale side' for the vertical display) and the HCL term. The HCL is in agreement for the right-handed/right hand case, but is in opposition for the left-handed left hand case. Thus there is a differential effect due to hand usage. Although the stereotype is not as strong when using the non-preferred hand, the lefthander now has a positive effect of HCL, whereas the righthander has a negative effect and hence has a lower stereotype strength. It is difficult, because of the left-hander living in a right-hand world to discriminate the effects of change of hands for the left-hander. The results are however clear for the right-hander using the preferred and non-

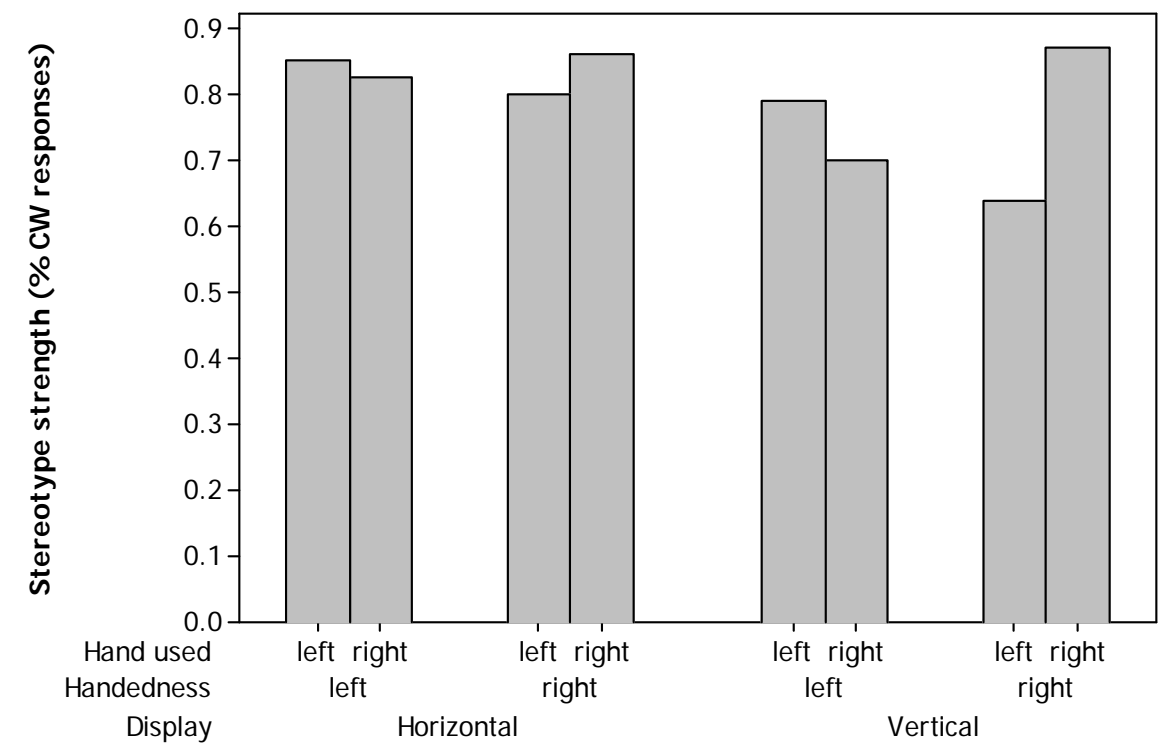

Fig. (3). Data of Chapanis and Gropper [10, figure 7, page 315] for left and right-handed subjects using their preferred and non-preferred hands. The display was either orientated horizontally or vertically; the control knob was always in the bottom position. 
preferred hands; the difference in the strength of the stereotype for the compatible arrangement of display and control is about $18 \%$ (from figure 7 of Chapanis and Gropper).

In summary, these two-dimensional arrangements of controls and displays show a significant effect of the location of the hand relative to the control, specifically the rotation direction of the control is modified somewhat by an expectancy to rotate the control in the direction in which the thumb points to the index finger. This is a 'natural' or more comfortable direction of rotation of the wrist or fingers. Lefthanders do not show a mirror image response to that of righthanders as they are also affected by the other principles that lead to the total stereotype strength (such as Warrick's principle and the scale-side principle). The response of lefthanders is however modified from that of right-handed persons by the difference in the hand/control location effect.

\section{(b) Three-Dimensional Display-Control Relationships}

Hand/control location effects can be seen in two different three-dimensional display/control arrangements:

Using left and right hands for control: In a display/control relationship when the right hand is used to rotate a control on the right hand side of a display, the natural tendency for movement is to move in the direction that the thumb points towards. This results in a clockwise rotation when viewed perpendicular to the control knob. When the left hand is used to rotate a control on the left-hand side of a display, the same tendency exists - motion is made towards the thumb direction. This results in an anticlockwise direction when viewed perpendicular to the control knob.

(ii) Using the same hand with controls in different locations: When a top and bottom control location is used with the right hand, the top location may result in a clockwise rotation and the bottom control may yield an anti-clockwise rotation when viewed perpendicular to the control knob. In this case, however, the direction of rotation that results may be dependent on the position of the control knob relative to the body of the controller and this would need to be taken into account in investigating stereotype strengths. Thus it is necessary to include this natural or preferred direction of rotation for various locations of a control relative to the body.

In both of the above cases, the resulting motions are dependent on body factors rather than the usual relationships between control rotation and resulting movement of the indicator along the scale. They are thus defined as 'Hand/Control Location' effects as they occur purely due to the design of control location relative to the body. Two examples of the use of Warrick's principle and the HCL effect are given in the following:

(i) A three-dimensional rotary control with linear display

This case is illustrated in Fig. (4). Engineering students commonly mention an implied linkage for this situation, as illustrated in Fig. (5). Again, this implied linkage is a rack and pinion mechanism connecting rotation of the knob to the linear motion of the rack (Fig. 5).

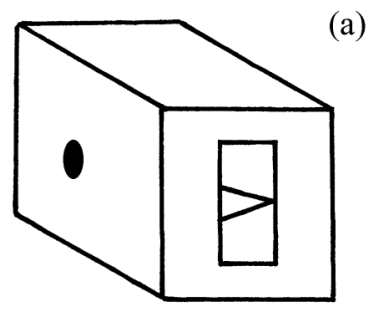

(a)

(b)

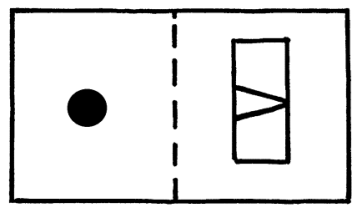

Fig. (4). (a) A three-dimensional display /control arrangement and (b) the 'unfolded' form used for analysis. This clearly shows the applicability of the Warrick principle in this case.

In the three-dimensional case of rotary controls with a linear display, the effect of the Warrick principle has been demonstrated in the data of [2]. In table 5 of that paper (page 211), data for three-dimensional control/display arrangements showed that Warrick's principle was the major contributor to the overall strength of the stereotype and the movements were reversible for decreasing scales. For scales that were on the front and top of the device and which were in the up and away directions, Warrick's principle accounted for $95 \%$ of the total stereotype strength. For scales that were on the front and top and which were in the left and right directions, it contributed $72 \%$ of the total stereotype strength.

When the control was on the right hand side of the device, Warrick's principle required a clockwise rotation of the control; when the control was on the left hand side, an anticlockwise rotation was required for the same indicator movement. Thus the left hand knob appeared to have a control reversal from the dominant clockwise rotation in order to move the indicator in the required direction. It was apparent that the $\mathrm{W}$ principle, being dominant, was the major factor in determining the direction of rotation of the control. In that case, subjects always used the right hand in control of the right-hand knob and the left hand when using the lefthand knob.

Two possible implied linkages are suggested for the case of a rotary control with linear display.

(a) The 'Unfolded' control/display relationship. Fig. (4a) shows a three-dimensional display/control device and (b) the same device where the system has been transformed into a two-dimensional arrangement by unfolding about the line dividing the planes of the control and the display. If the device is analysed in this form as a two-dimensional control/display relationship, it is seen that the Warrick principle is operational and that anticlockwise rotation is expected for the left-hand control and clockwise rotation for the right-hand control. This is fully in accord with the data of Hoffmann [2].

(b) A three-dimensional linkage. In a three-dimensional form, this 'implied linkage' may be viewed as a single shaft passing through the device, having a pinion attached to the shaft directly below the display (Fig. 5). Attached to the indicator is a rack that meshes with the pinion. Again, this arrangement gives the anticlockwise rotation for the left-hand control and clockwise rotation for the right-hand control. 


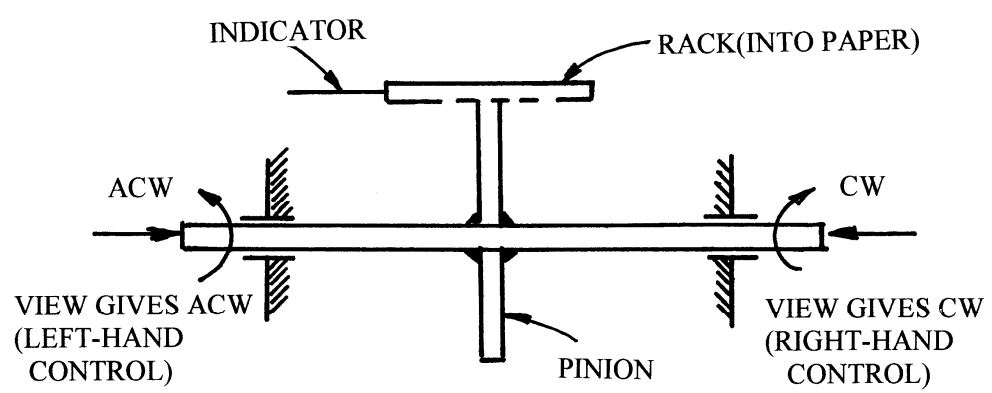

Fig. (5). A possible form of 'implied linkage' for the three-dimensional display/control arrangement shown in Fig. (4), when the rotary control is either on the left or right-hand side of the display (Front view).

For this display/control arrangement, the postulated HCL term, if written into an expression to determine component stereotype strengths [2] would yield a result identical to the inclusion of Warrick's principle when using the unfolded control/display arrangement. This is so, as all subjects when performing these tests used the right hand on the right-hand control and the left hand when using the left-hand control. The dominant hand movement was then in the direction pointed by the thumb to the first finger. Thus it is not possible with that data set to discriminate between these two possible explanations (HCL and Warrick) for the apparent reversal of control rotation (anticlockwise for the left hand and clockwise for the right hand).

(ii) A three-dimensional display control relationship. Rotary controls in top and bottom locations with a circular display.

Of particular interest are the recent results of Chan and Chan [11,12] who have experimentally established stereotypes for circular displays when there are controls in different planes. Here the case where there is a circular display on the front face of the device and controls located to the right and top [11] or left and bottom [12] of the device, are considered. A not-to-scale diagram of the relative location of display and controls is shown in Fig. (6). It should be noted that the subject groups were different in the two experiments reported in these two papers and hence there may be effects due to these different groups. The data are given in Table $\mathbf{1}$.

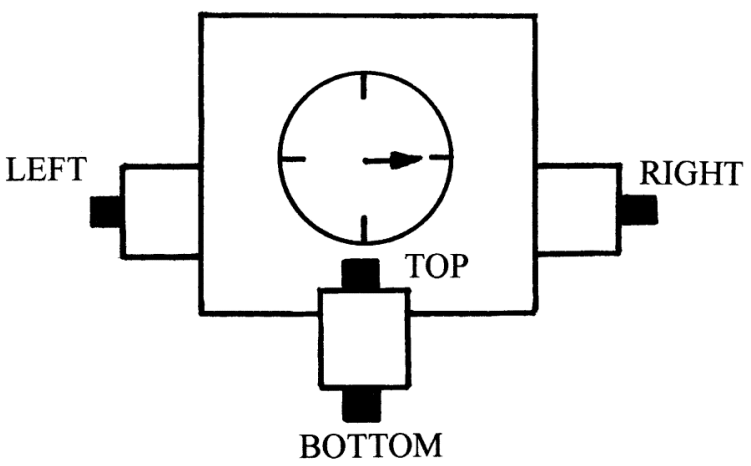

Fig. (6). Locations of the controls relative to the display in the experiments of $[11,12]$. Subjects sat in front of the CRT on which the circular display was presented. Top and right rotary controls are from [11]; Left and Bottom rotary controls are from [12]. Drawing is not to scale.
Table 1. Proportion of Clockwise Rotations for Instructed Clockwise Movement of the Indicator. Data of Chan and Chan $[11,12]$. Top, Bottom, Right-Hand and Left-Hand Refer to the Location of the Control Relative to the Display

\begin{tabular}{|c|c|c|c|c|}
\hline $\begin{array}{c}\text { Indicator Position } \\
\text { (O'Clock) }\end{array}$ & $\begin{array}{c}\text { Top } \\
{[\mathbf{1 1}]}\end{array}$ & $\begin{array}{c}\text { Bottom } \\
{[\mathbf{1 2}]}\end{array}$ & $\begin{array}{c}\text { Right-Hand } \\
{[\mathbf{1 1}]}\end{array}$ & $\begin{array}{c}\text { Left-Hand } \\
{[\mathbf{1 2}]}\end{array}$ \\
\hline \hline 12 & .947 & .474 & .921 & .158 \\
\hline 3 & .947 & .342 & .816 & .158 \\
\hline 6 & .942 & .237 & .763 & .237 \\
\hline 9 & .947 & .342 & .895 & .316 \\
\hline
\end{tabular}

There are several features apparent in these data:

(a) Top and right-hand controls give a strong clockwise response

(b) Bottom and left-hand controls give an anticlockwise response, which is not as strong as that for the other control locations.

(c) The differences in these columns of data suggest reinforcing or opposing effects of different principles.

These results are essentially in agreement with the data for engineering students mentioned earlier in that there appears to be a dominant form of response, which is clockwise or anticlockwise depending on the location of the control. The results are suggestive of the presence of a Warrick-type principle or one involving HCL. If it is a Warrick principle effect, the data may be biased due to the fact that engineering students were used as subjects; if an HCL effect, the data may be generalised to other subject groups.

This raises the question as to whether there is the possibility of an 'implied linkage' as found for the application of Warrick's principle in the two-dimensional and threedimensional cases with linear scales. A possible linkage is shown in Fig. (7).

In this linkage, there is a single shaft passing through between the left and right-hand controls (or the top and bottom controls). This is as in the case of linear scales (Fig. 5). Attached to the shaft is a bevel gear. This meshes with a bevel gear on the shaft carrying the indicator. In this case, an 


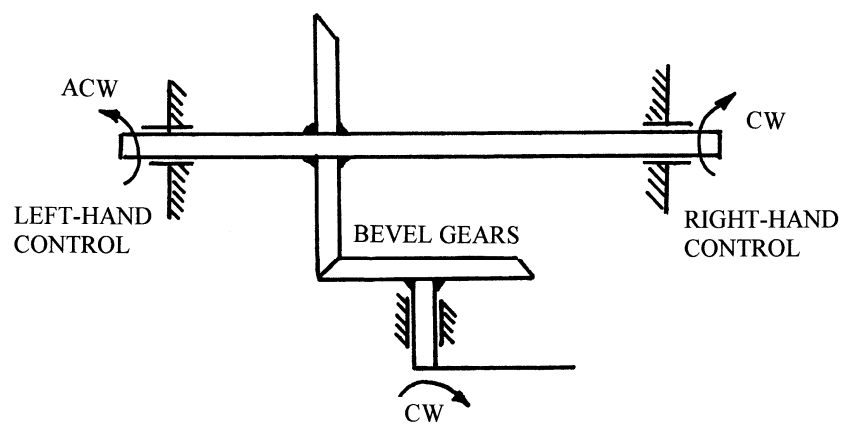

Fig. (7). A possible 'implied linkage' for the three-dimensional display/control relationship studied by Chan and Chan [11,12] with a circular display on the front face and rotary controls on the left and right-hand sides (Plan view).

anticlockwise rotation of the left-hand control produces a clockwise rotation of the indicator. Also, a clockwise rotation of the right-hand control produces the same clockwise rotation of the indicator. Similar reasoning produces the results found for the top and bottom control locations.

Thus, it appears that there may be three-dimensional equivalents to the two-dimensional case. It is therefore possible that biases are introduced into the data by testing only engineers who have a mental model of the way in which devices operate. This requires further investigation, as it is not known whether engineering subjects have any mental model of an 'implied linkage' in this more complex case. It should be noted that the data cannot be accounted for by the Clockwise-Clockwise (CC) principle suggested by Chan and Chan $[11,12]$ as the principle does not account for the control reversal, but requires a separate set of relationships for each control location. Either of the implied Warrick linkage or the HCL effect may account for such a reversal. The case for HCL is presented in the following analysis. Here it will be seen that the Warrick effect is small and the dominant effect is that due to hand/control location effects.

The approximate locations of controls used by Chan and Chan $[11,12]$ are illustrated in Fig. (6). Note that Chan and Chan [11] investigated the right and top control locations (as well as other locations) and Chan and Chan [12] reported data on the left and bottom control locations. In this analysis the 'unfolded' display/control arrangements for the top, bottom, left and right-hand controls have been used. Along with this unfolded arrangement the 'linearised' circular display (Chan, Courtney and So, [13]) is used in order to introduce the Clockwise for clockwise (CC), Scale side (SS) and Warrick (W) principles. An example of these for the 12 and 3-o'clock locations are shown in Fig. (8).

Expressions for the contribution of the various principles to the total stereotype strength have been developed by the method introduced by Hoffmann [2], which assumes that the effects of each of the principles are linearly additive [2]. For example, in Fig. (8a), in which there is a left-hand side control with the front face circular display and the indicator is at the 12-o'clock position (shown in the 'unfolded' arrangement), the scale-side principle would produce a clockwise rotation of the display along with the clockwisefor-clockwise principle. Here the HCL effect would be predicted to produce an anticlockwise rotation of the display, as on the left-hand side of the device, the 'natural' direction of rotation of the control is anticlockwise. Adding these components yields equation 1, with the further 0.5 being for the chance probability of a clockwise or anticlockwise rotation of the control [2]. The value .158 arises from the experimentally-measured total stereotype strengths given in Table 1.

Table 2 gives the full breakdown of components of the stereotypes for each of the sixteen combinations of display position $(12,3,6$, and 9 o'clock) at each of the four control locations. As the direction of motion of the control is dependent on the grip of the knob used by the subjects, in this analysis the grip shown in Fig. (9a) has been assumed.

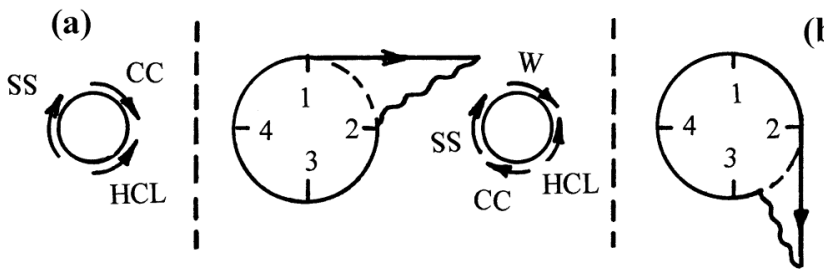

(b)

Fig. (8). The 'linearised' circular display for the unfolded left-hand control, front-face display arrangement. Arrows show the direction of control rotation predicted by the various principles and the hand/control location effect. (a) is for the display in the 12-o'clock position; (b) is for the display at the 3-o'clock position.

The equations 1 to 16 have been solved by the method of Hoffmann [2]. For example, for the case of left and right-hand controls, equations 1 to 8 can be simply added to solve for $(\mathrm{CC}+\mathrm{SS})$. Subtraction of equations 5 to 8 from equations 1 to 4 will yield a value for HCL, and so on. Note that the $\mathrm{CC}$ and $\mathrm{SS}$ components are always additive in these equations and the effects cannot be separated.

Left and Right-hand controls: Solution of equations 1 to 8 yields $\mathrm{HCL}=.316,(\mathrm{CC}+\mathrm{SS})=.033$ and $\mathrm{W}=-.02$. This solution accounts for $74 \%$ of the stereotype. The dominant term is seen to be HCL, with, in this form of analysis, the W term being negligible. The maximum error of fit to the eight data results is .079 , with an average error of .059 .

Top and Bottom controls: Solution of equations 9 to 16 yields $\mathrm{HCL}=.299,(\mathrm{CC}+\mathrm{SS})=.147$ and $\mathrm{W}=.058$. The sum of magnitudes in this case adds to .50 , thus accounting for $100 \%$ of the stereotype strength. The maximum error of fit to the data is .068 , with an average error of .032 . 
Table 2. Analysis of the Chan and Chan [11,12] Data for Stereotype Components Using the Hand/Control Location (HCL) Effect. Control Locations are as Shown in Fig. (6), Indicator Positions are Based on Clock Locations

\begin{tabular}{|c|c|c|c|}
\hline $\begin{array}{c}\text { Control } \\
\text { Location }\end{array}$ & $\begin{array}{l}\text { Indicator } \\
\text { Position } \\
\text { (O'Clock) }\end{array}$ & Describing Relationship & $\begin{array}{c}\text { Equation } \\
\text { Number }\end{array}$ \\
\hline \multirow{4}{*}{ Left } & 12 & $-\mathrm{HCL}+\mathrm{CC}+\mathrm{SS}+.5=.158$ & 1 \\
\hline & 3 & $-\mathrm{HCL}+\mathrm{CC}+\mathrm{SS}+\mathrm{W}+.5=.158$ & 2 \\
\hline & 6 & $-\mathrm{HCL}+\mathrm{CC}+\mathrm{SS}+.5=.237$ & 3 \\
\hline & 9 & $-\mathrm{HCL}+\mathrm{CC}+\mathrm{SS}-\mathrm{W}+.5=.316$ & 4 \\
\hline \multirow{4}{*}{ Right } & 12 & $\mathrm{HCL}+\mathrm{CC}+\mathrm{SS}+.5=.921$ & 5 \\
\hline & 3 & $\mathrm{HCL}+\mathrm{CC}+\mathrm{SS}-\mathrm{W}+.5=.816$ & 6 \\
\hline & 6 & $\mathrm{HCL}+\mathrm{CC}+\mathrm{SS}+.5=.763$ & 7 \\
\hline & 9 & $\mathrm{HCL}+\mathrm{CC}+\mathrm{SS}+.5=.895$ & 8 \\
\hline \multirow{4}{*}{ Top } & 12 & $\mathrm{HCL}+\mathrm{CC}+\mathrm{SS}-\mathrm{W}+.5=.947$ & 9 \\
\hline & 3 & $\mathrm{HCL}+\mathrm{CC}+\mathrm{SS}+.5=.947$ & 10 \\
\hline & 6 & $\mathrm{HCL}+\mathrm{CC}+\mathrm{SS}+.5=-.942$ & 11 \\
\hline & 9 & HCL-CC-SS $+.5=.947$ & 12 \\
\hline \multirow{4}{*}{ Bottom } & 12 & -HCL-CC+SS-W+.5 = .474 & 13 \\
\hline & 3 & $-\mathrm{HCL}+\mathrm{CC}+\mathrm{SSt} .5=.342$ & 14 \\
\hline & 6 & $-\mathrm{HCL}+\mathrm{CC}+\mathrm{SS}-\mathrm{W}+.5=.237$ & 15 \\
\hline & 9 & $-\mathrm{HCL}+\mathrm{CC}+\mathrm{SS}+.5=.342$ & 16 \\
\hline
\end{tabular}

All cases: Here the fit to the sixteen equations gives HCL $=.308,(\mathrm{CC}+\mathrm{SS})=.09$ and $\mathrm{W}=.019$. These account for $83 \%$ of the total stereotype strength. The errors are however increased as there are differences between components of the stereotypes for the two cases of left/right and top/bottom controls (possibly due to the two different subject groups). The maximum error of fit is .173 and the mean error is .07 .

\section{DISCUSSION AND CONCLUSIONS}

Much of the early research on handedness and hand used, as reviewed here, has been ignored in later literature. This is unfortunate as there are obviously features in these sets of data that show a significant effect on stereotype strength. Most of the later research has concentrated on using righthanded subjects in order to eliminate effects of handedness. All two-dimensional research has then been performed using the right hand for making control responses. It is usually only when three-dimensional arrangements are studied that the effects of handedness and hand used become strongly relevant to the researcher. In three-dimensional arrangements, it is more often necessary for the subject to use the nonpreferred hand due to the location of the body relative to the controlled device.

This paper has investigated the hypothesis that there is an effect of the hand grip and the hand used on the component strengths of stereotypes for display-control relationships and, consequently, on the overall strength of the stereotype. Support for the hypothesis is found in data from two- and three-dimensional arrangements of displays and controls. In some arrangements, the HCL hypothesis produces results identical to the Warrick principle. In the most complex case investigated, the HCL effect is seen to outweigh the effects of the Warrick principle. The HCL effect is, however, more generally applicable than any of the principles affecting component strengths of stereotypes, as it is simply dependent on the hand used and grip used for moving the control knob.

The importance of the HCL vs Warrick effects lie in the consequences: if Warrick is dominant, there may be effects of the subject group as engineers, in particular through knowledge of mechanisms, have developed 'mental models' and 'implied linkages' that may bias the way in which they respond. Others, without this knowledge may respond quite differently. This difference is seen in the data of Hoffmann [2] for two-dimensional control-display arrangements.

It appears from the analysis of this paper that, in the three-dimensional cases considered, the HCL effects may be dominant. In other words, the hand used and the posture of the hand in making the control action is of greater importance than the Warrick effect. The situation for the two-dimensional arrangements is quite different. Here there may be a significant effect of the HCL, but this is in competition with other principles that override the effects. When there is no display present (ie when turning a knob to increase brightness of a light) the hand-used effect is very strong.

The above analysis of the Chan and Chan [11,12] data has demonstrated two points:

there is the possibility of 'implied linkages' in three-dimensional display/control relationships that may bias experimental results when engineering students are used as subjects. The research that has been reported for three-dimensional arrangements needs to be repeated with non-engineering subjects in order to see if the effects of Warrick's principle apply in those cases - and also if the HCL model may be applicable.

(b) it appears necessary to introduce a 'Hand/Control Location' effect in order to account for the data found for stereotypes in three-dimensional display/control arrangements. It is possible that these effects of hand and control location totally account for what may appear to be the Warrick components found in earlier research [2] on rotary controls with linear displays in three-dimensional arrangements.

Further experiments are required in order to separate the effects of the $\mathrm{W}$ and HCL in those arrangements where there is an apparent reversal of control rotation. These experiments would involve the use of the same hand when reaching to a control on the opposite side of a display or using the other hand when rotating a control that is either on the top or bottom of a display. In each case it would be necessary to record the posture of the hand in performing the control action. For example, in the case of the bottom control in Fig. (6), it is possible to hold the knob in two different hand postures (Fig. 9). The more comfortable posture, requiring little wrist rotation, would be compatible with the HCL effect. The less comfortable hand posture, requiring considerable wrist rotation would not be consistent with the HCL effect used in developing the equations for component stereotype strengths. As the HCL effect postulates a 'natural' 


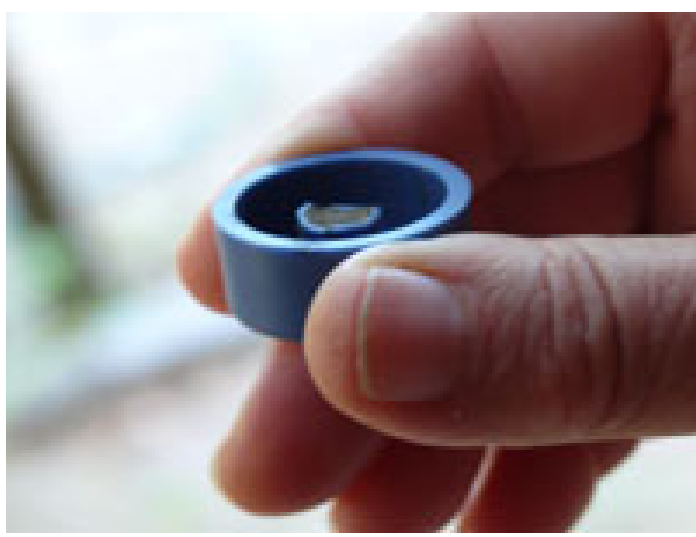

(a)

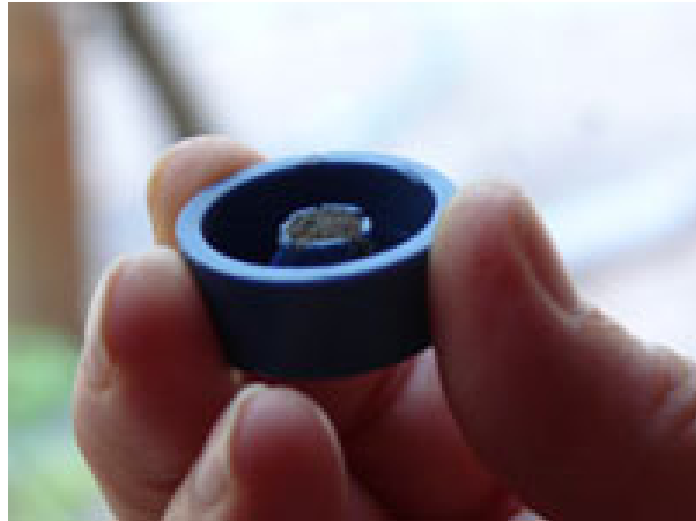

(b)

Fig. (9). Illustration of two different grips/hand postures that may be used for rotating a bottom knob as shown in Fig. (6). When viewed from the bottom of the knob (a), according to the HCL postulate, will produce an anticlockwise rotation; grip (b) would produce a clockwise rotation. The type of grip is likely to be dependent on the height of the knob relative to the body.

rotation towards the thumb pointing direction, the use of the left hand in the display/control arrangements used by Chan and Chan $[11,12]$ is necessary to establish the validity of the HCL effect with top/bottom controls - and here the hand grip/posture to be used would need to be specified.

It was noted that the data from Chan and Chan was for two different subject groups. This is likely to have added some uncertainty to the number of clockwise responses for each of the control conditions. The fact that the HCL proposal comes out so strong in the analysis, even with different groups, seems to indicate that this is a valid concept.

\section{ABBREVIATIONS}

$\mathrm{CC}=$ The 'clockwise-for-clockwise' principle suggested by Chan and Chan $[11,12]$ when there is a rotary control with a circular display. The expectancy with this principle is that a clockwise rotation of the control will produce a clockwise rotation of the indicator on the display

$\mathrm{CR}=$ The stereotype principle where a clockwise rotation of a rotary control is expected to produce a movement of the indicator to the right

HCL $=$ The postulated physical principle developed in this paper, where there is an expectancy that a control will be rotated in a direction determined by the grasp of the control, but in a direction from the thumb to the tip of the index finger

$\mathrm{SS}=$ The scale-side principle of Brebner and Sandow [3]. This may be stated as the indicator will move in the direction of the side of the control that is on the same side of the knob as the scale is on the side of the display
$\mathrm{W}=$ Warrick's principle which states that the indicator is expected to move in the same direction as that part of the control nearest to the display

\section{REFERENCES}

[1] Warrick MJ. Direction of movement in the use of control knobs to position visual indicators. USAF AMC 1947; report No. 694-4C.

[2] Hoffmann ER. Strength of component principles determining direction of turn stereotypes - linear displays with rotary controls. Ergonomics 1997; 40: 199-222.

[3] Brebner J, Sandow B. Direction-of-turn stereotypes--conflict and concord. Appl Ergon 1976; 7(1): 34-6.

[4] Worringham CJ, Berringer DB. Operator orientation and compatibility in visual-motor task performance. Ergonomics 1989; 32(4): 387-99.

[5] Worringham CJ, Berringer DB. Directional stimulus-response compatibility: a test of three alternative principles. Ergonomics 1998; 41(6): 864-80.

[6] Verhaegen P, Bervoets R, Debrabandere G, et al. Direction of movement stereotypes in different cultural groups. In: Chapanis A, Ed. Ethnic Variables in Human Factors Engineering. Baltimore: The John Hopkins University Press 1975; pp. 135-149.

[7] Bradley JV. Direction-of-knob-turn stereotypes. J Appl Psychol 1959; 43(1): 21-4.

[8] Holding DH. Direction-of-motion relationships between controls and displays moving in different planes. J Appl Psychol 1957; 41: 93-7.

[9] Holding DH. The effect of initial pointer position on displaycontrol relationships. Occup Psychol 1957; 31: 126-30.

[10] Chapanis A, Gropper BA. The effect of the operator's handedness on some directional stereotypes in control-display relationships. Hum Factors 1968; 10: 303-20.

[11] Chan WH, Chan AHS. Movement compatibility for rotary control and circular display - computer simulated test and real hardware test. Appl Ergon 2003; 34: 61-71.

[12] Chan AHS, Chan WH. Movement compatibility for circular display and rotary controls positioned at peculiar positions. Int J Ind Ergon 2006; 36: 737-45.

[13] Chan AHS, Courtney AJ, So KWY. Circular display with thumbwheels: Hong Kong preferences. Int J Hum Factors Ergon Manuf 2000; 10(4): 453-63. 